\title{
François Jacqmin, auteur comique
}

\section{Laurent ROBERT}

François Jacqmin l'a affirmé lui-même, en clausule de Camera oscura ${ }^{1}$ : « Je suis un auteur comique !». L'exclamation tient moins du paradoxe que de l'exhortation : quoi qu'il en soit du ton de gravité de certains de ses poèmes ou de la dimension métaphysique de son œuvre, le poète des Saisons se voulait, se serait voulu «un auteur comique ». Plusieurs déclarations le confirment. Au terme d'un entretien accordé en 1985 à Pascal Goffaux, il notait :

[...] ce qui me dérange très fort dans l'interview, c'est que cela m'oblige à me prendre au sérieux. Ce que je ne suis pas du tout. En fait, j'aurais souhaité être un auteur comique plutôt qu'un auteur sérieux, mais je n'ai pas le génie du comique et peut-être pas le génie du sérieux non plus ${ }^{2} \ldots$

Et lors des conférences prononcées dans le cadre de la Chaire de Poétique de l'Université Catholique de Louvain-la-Neuve, il se faisait plus explicite encore ${ }^{3}$ :

L'inclinaison que j'ai pour le burlesque aurait pu faire de moi un auteur comique. Ce penchant se manifeste d'une manière souterraine, prenant davantage le ton et la tournure de l'ironie. Quant à mon attitude littéraire proprement dite, à ce sujet, il m'est souvent apparu que les choses s'entendent mieux lorsqu'elles sont enrobées d'humour. L'absurde, l'ironie, la fantaisie, me paraissent des voies intellectuelles éminemment praticables.

C'est précisément à cette «manière souterraine» qu'est l'ironie que je souhaite ici m'attacher. Mon approche ne se veut pas seulement sémiologique: étudier l'ironie chez François Jacqmin, ce n'est pas uniquement décrire l'usage d'une figure de rhétorique ; c'est surtout mettre au jour les réticences du poète, ses rejets, ses défiances moqueuses, et c'est s'aventurer dans les lieux où l'œuvre prend des risques, où elle n'est plus loin de l'aporie.

\section{Modèle de description}

S'il n'entre pas dans mon propos d'instruire à nouveau la question d'une définition de l'ironie ${ }^{4}$, il convient toutefois de préciser le sens que prendra cette notion dans les pages qui vont suivre. La tradition rhétorique a souvent confondu l'ironie et l'antiphrase. Ainsi en va-t-il de la définition de Fontanier selon qui l'ironie consisterait à «dire, par une raillerie, ou

\footnotetext{
${ }^{1}$ Camera oscura fut publié une première fois en 1976 aux Éditions Temps Mêlés. Je cite à partir de la réédition des Éditions de la Différence (La Rose de décembre et autres poèmes. Paris, Éditions de la Différence, coll. Clepsydre, 2000, p.66)

${ }^{2}$ Pascal Goffaux et François Jacqmin, Parole gelée. Gerpinnes, Éditions Tandem, coll. Alentours, 2006, p.19.

${ }^{3}$ François Jacqmin, Le poème exacerbé. Louvain-la-Neuve, Presses Universitaires de Louvain UCL, coll. Chaire de Poétique 6, 1992, p.25. Voir également une interview pour le magazine ActuaPress : «La notion de comique est capitale pour moi. [...] De temps en temps, la poésie devrait faire rire ou sourire, d'autant qu'il y a une véritable force dans le comique... On sait que les pouvoirs politiques ou religieux en veulent au comique. Le tyran en l'homme sent ce que constitue l'arme du comique et l'artiste aujourd'hui a tendance à l'oublier. Je souhaiterais n'avoir écrit que des choses très comiques » (Martine Gerardy, "Qu'adviendrait-il du monde si François Jacqmin était fidèle à sa promesse ? » in ActuaPress n²87B. 23 novembre 1989, p.10). Au terme d'une brève séquence de poèmes peu connus, François Jacqmin note encore que « [L'infini] est à l'origine de notre sentiment du comique » («Contribution à un manuel de savoir vivre à l'usage des gens du monde anxieux au sujet de l'infini », in Aa Revue n ${ }^{\circ} 188.25$ juin 1988, p.14).

${ }^{4}$ Sur ce point, voir notamment Philippe Hamon, L'Ironie littéraire. Essai sur les formes de l'écriture oblique (Paris, Hachette, coll. Recherches littéraires, 1996).
} 
plaisante, ou sérieuse, le contraire de ce qu'on pense, ou de ce qu'on veut faire penser ${ }^{5} »$. Si l'aspect de « raillerie » - ou d'éthos moqueur - n'est pas remis en cause par les sémioticiens contemporains, plusieurs auteurs ont néanmoins jugé cette définition trop restrictive. Conformément à l'étymologie, l'ironie constituerait davantage une «interrogation ${ }^{6}$ »; elle mettrait en scène pour le lecteur un conflit de sens, voire un conflit de «valeurs ${ }^{7}$ ». Par le biais de l'ironie, ce sont des échelles de valeurs qui sont représentées et, partant, des systèmes de règles ou de normes qui sont contestés. En outre, l'énoncé ironique ne serait pas univoque, mais polysémique : même si l'on s'en tient à une structure binaire - comme dans l'antiphrase -, «l'encodeur, quand il énonce $\mathrm{x}$, ne renonce pas à nous faire entendre $\mathrm{x}$ en même temps que non- $x^{8} »$. Enfin, Sperber et Wilson voient dans l'ironie une «mention », autrement dit un processus citationnel ou métacommunicationnel, par lequel tout énoncé ironique reposerait, de façon le plus souvent implicite, sur une double énonciation ${ }^{9}$. J'expliciterai cet aspect de l'ironie - et illustrerai les autres par la même occasion - en recourant à un exemple pris dans l'œuvre de François Jacqmin. Dans Le Domino gris, une section s'intitule « Art poétique ». À bon droit, le lecteur pourrait s'attendre à y découvrir des précisions sur l'art d'écrire ainsi, éventuellement, que leur application dans le texte qui les énonce. Il y lira plutôt des aphorismes glacés, où l'art se voit frappé d'une condamnation morale :

Tout art est un mensonge ajouté à la matière.

L'œuvre est le fait des scélérats de la fiction.

Pour être, l'être a décliné à tout jamais ce qui pouvait le concevoir.

Le désert est une pierre qui s'indigna de la forme ${ }^{10}$.

L' «Art poétique » de François Jacqmin est un refus de l'art. Les textes qui le composent fixent le titre en citation. L'énonciation «Art poétique» est incluse dans une autre énonciation qui dirait, par exemple, que «d'aucuns prétendent écrire un "art poétique", alors que tout art est un mensonge ajouté à la matière ». Intituler un ensemble de textes «Art poétique », c'est non seulement convoquer la notion classique d'art poétique ; c'est aussi user de ces termes comme d'une citation - citation qui devient ironique une fois qu'ont été lus les quatre poèmes qui composent cette section. Et le sens - ou la valeur - de ce qui est ironisé n'est pas non plus univoque: si François Jacqmin met en doute la validité de l'art et la pertinence d'un art poétique, son «Art poétique »n'en existe pas moins - si ironique et lapidaire soit-il ${ }^{11}$.

\section{Le langage en procès}

L'«Art poétique» n'est pas le seul titre à partir duquel François Jacqmin sème la contradiction. Dans Le Domino gris toujours, l'intitulé «Gradus ad Parnassum » induit une autre déclinaison d'un art poétique. Le «Gradus ad Parnassum »- littéralement « escalier

\footnotetext{
${ }^{5}$ Cité par Bernard Dupriez (Gradus. Les procédés littéraires (Dictionnaire). Paris, UGE, coll. 10/18, 1984, p.265).

${ }^{6}$ Dupriez, ibid.

${ }^{7}$ Hamon, op.cit., p. 28.

${ }^{8}$ Groupe $\mu$, «Ironique et iconique » in Poétique $\mathrm{n}^{\circ} 36$. Novembre 1978, p.427.

${ }^{9}$ Dan Sperber et Deirdre Wilson, «Les ironies comme mention », in Poétique ${ }^{\circ} 36$. Novembre 1978, pp.399412. Cf. aussi Groupe $\mu$, op. cit., p.434. L'hypothèse de l'ironie comme processus métacommunicationnel, sinon intertextuel, est également admise par Hamon (op. cit., p.152).

${ }^{10}$ François Jacqmin, Le Domino gris. La Louvière, Le Daily-Bul, 1984. L'ouvrage est dépourvu de pagination.

${ }^{11}$ Comme l'indiquait du reste déjà Jean Paulhan à propos de la poésie moderne, « nos arts littéraires sont faits de refus »; ce serait même leur seule façon d'encore exister (Les Fleurs de Tarbes ou La Terreur dans les Lettres. Paris, Gallimard, coll. Folio Essais n 147,1990 [1941], p.41).
} 
vers le mont Parnasse », séjour des Muses - ne réfère pas à autre chose, à l'Époque Classique, qu'aux figures de rhétorique même. C'est par la maîtrise des moyens rhétoriques que le poète peut atteindre le sublime. Un «gradus » désigne donc un répertoire de figures, un manuel de composition littéraire ${ }^{12}$. Or, le «Gradus ad Parnassum» de François Jacqmin ne propose d'autre recette que celle de la retenue, voire de l'abstention :

Il n'est pas contraire au bon goût de vivre sans métaphores.

$\mathrm{Ne}$ rien dire aide l'esprit à ne pas conclure tout en retenant sa distinction.

Il est en bon chemin celui qui ne choisit ni l'image ni le verbe.

Il faut se garder de dénigrer ce qui est sans figure : l'inexprimable y engrange des merveilles.

Avec «Le Tombeau de Linné ${ }^{13} »$, ce n'est plus l'écriture qui est visée, à travers la condamnation de l' «image », du «verbe » ou des «métaphores »; c'est la nomination même qui se voit disqualifiée. De même que celle de l'art poétique ou que celle du gradus, la composition d'un «tombeau » est une pratique d'écriture codifiée. Écrire un «tombeau», c'est, pour un poète, rendre hommage à un pair, proche ou lointain dans le temps. Et c'est très souvent unir, dans les mêmes vers ou les mêmes pages, exercice d'admiration et programme poétique. Pour le poète contemporain francophone, la référence en la matière est sans aucun doute Mallarmé, auteur des «Tombeau d'Edgar Poe», «Tombeau de Charles Baudelaire » et «Tombeau [de Paul de Verlaine] ». De son côté, François Jacqmin contrevient une première fois à la pratique littéraire du «tombeau » en vouant ses courtes proses non à un poète, non à un écrivain, mais à Carl von Linné, père de la nomenclature binominale utilisée en botanique et en zoologie ${ }^{14}$. Et il le fait d'autre façon en rendant hommage non à la précision scientifique mais à «l'univers impensé », et en célébrant «le coquelicot absolu » qui « a pour propriété de protéger la méditation des affronts de la raison ». Il nomme ce qui ne peut se nommer, la «fleur qui se définit sans un mot » et qu' «à défaut d'autres précisions, on [...] appelle, très imparfaitement, "la fleur de l'avalanche tranquille" ». Avec "Le Tombeau de Linné », François Jacqmin n'ironise donc pas seulement sur le recours à un sous-genre littéraire déterminé ; il met tout autant en cause la prétention même à dire. Une attitude similaire se fait jour dans le recueil posthume Éléments de géométrie, à cette nuance près cependant que l'impertinence de la nomination y est analogue de l'impertinence de la représentation picturale ou architecturale ${ }^{15}$. Le titre, qui pourrait convenir à un traité ou à un manuel, n'en est pas moins ironique, puisque les poèmes invitent surtout à désapprendre et à s'abstenir de rien esquisser : «L'âme tient la géométrie pour une dégradation.// Dans le cercle autant que dans la pyramide, elle retrouve l'exécrable arrière-goût de l'axiome. »

L'ironisation de la capacité à représenter, par quelque moyen que ce soit, ne repose pas uniquement sur la tension manifeste entre des textes et le titre qui les chapeaute. Nombre de poèmes de François Jacqmin constituent par eux-mêmes de petites bombes ironiques. Dans Les Saisons particulièrement, plusieurs textes s'amusent des velléités de (re)motivation du langage par le biais de la création poétique. À la différence d'un Francis Ponge notamment,

\footnotetext{
${ }^{12}$ C'est en souvenir de ces «gradus » passés que Bernard Dupriez intitula lui aussi son répertoire de figures Gradus (op.cit.).

${ }^{13}$ Le Domino gris, op. cit.

${ }^{14}$ François Jacqmin est aussi l'auteur d'un curieux « Tombeau de Molière », conférence dont le manuscrit a été publié dans Aa revue, revue de l'écrivain liégeois Richard Tialans (Aa Revue ${ }^{\circ} 198,19$ octobre 1989, pp. 2-19). Le poète ne s'y départit pas de son souci de ne pas conclure, de ne jamais affirmer ni trancher : «Nous avons considéré que le Don Juan de Molière constitue un exemple de cette liberté de nous placer au-delà ou en deçà de toute conclusion. Nous voyons combien cette situation peut être fertile, en ce qu'elle nous empêche de classer, c'est-à-dire de disqualifier. » (p.3).

${ }^{15}$ François Jacqmin, Éléments de géométrie. Soumagne, Tétras Lyre, coll. Lyre sans bornes, 2005. Recueil non paginé.
} 
François Jacqmin ne croit pas que la poésie puisse, pour reprendre l'expression de Mallarmé, « rémunérer le défaut des langues »- qu'elle puisse, en d'autres termes, corriger l'arbitraire du signe et que, par l'imagination, par la fantaisie, par la rêverie linguistique, elle puisse rendre un mot adéquat à l'objet qu'il désigne ${ }^{16}$. Il accepte néanmoins de feindre de jouer le jeu, le temps d'un poème :

Dans toutes les langues du monde, l'œuf est un mot en formation.

C'est un gousset d'aspirations molles retenues par une frontière de silence chaulé.

Le temps le tient dans sa mire.

Sa première tentative d'être sera une cassure; son expression, une astuce au visage de poussin ${ }^{17}$.

Dans ce poème du «Printemps », c'est bien à une rêverie cratyliste ou mimologiste - pour reprendre la terminologie de Genette ${ }^{18}$ - que François Jacqmin semble se livrer. Si la langue première, originelle, a disparu, si elle a éclaté en une multitude d'idiomes distincts, il n'en demeure pas moins des traits communs à toutes les langues qu'il revient au poète de déceler et de mettre au jour - puisque le linguiste, pour sa part, semble y avoir renoncé. La première phrase du poème admet ce principe d'universalité, déjà présent dans le Cratyle de Platon ${ }^{19}$. Et, comme la plupart des cratylistes, c'est d'un mot seul que François Jacqmin s'attache à produire la motivation ${ }^{20}$. En l'occurrence, c'est «l'œuf » dont il faudra montrer qu'il est de toute évidence « un mot en formation », puisqu'il est en même temps - par excellence et par définition - un être en formation. La courte description qui suit se devra dès lors d'être équivoque, jouant sur la polysémie de termes qui peuvent receler des connotations aussi bien existentielles ou matérielles que métalinguistiques. Finaud, François Jacqmin ne recourt, dans la deuxième phrase, qu'à deux termes pour évoquer le mot « œuf » : le «silence », notation métalinguistique très générale et, en quelque sorte, minimale; et les «aspirations », terme qui peut se rencontrer en phonétique ${ }^{21}$. Les deux termes sont insérés dans deux métaphores dont la première décrit l'œuf-être en formation («gousset d'aspirations molles ») et la seconde sa coquille («frontière de silence chaulé »). C'est là tout l'effort mimologiste de François Jacqmin ; il n'en fera pas plus. Le mot « œuf » est une «aspiration » cernée de «silence »rien d'autre. En fait, il n'y a rien à dire du mot «œuf », comme il n'y a, pour François Jacqmin, rien à dire des mots : ils sont là, c'est tout, il faut s'en satisfaire si l'on est contraint de les utiliser. La démonstration que «l'œuf est un mot en formation » est par conséquent volontairement boiteuse. Par la faible consistance des notations métalinguistiques, elle tient pratiquement de la pétition de principe : le mot « œuf », «dans toutes les langues du monde »,

\footnotetext{
${ }^{16}$ Gérard Genette voit dans les nombreuses réflexions et créations sur le caractère arbitraire ou motivé du langage un véritable genre littéraire, qui traverse les siècles depuis le Cratyle de Platon jusqu'à la littérature contemporaine. On consultera évidemment sa somme (Mimologiques. Voyage en Cratylie. Paris, Le Seuil, coll. Points Essais n³86, 1999).

${ }^{17}$ François Jacqmin, Les Saisons. Bruxelles, Phantomas, 1979, p.23.

${ }^{18}$ Genette propose de «baptiser cratylisme (ou mimologisme) secondaire [...] le désir presque irrésistible [...] de corriger d'une manière ou d'une autre cette erreur du nomothète que Mallarmé appelle le "défaut des langues"et donc d'établir ou rétablir dans le langage, par quelque artifice, l'état de nature que le cratylisme "primaire", celui de Cratyle, croit naïvement y voir encore ou déjà établi » (op. cit., p.40).

19 «Cratyle ici présent déclare [...] qu'une certaine rectitude de dénomination existe originellement [...] et la même pour tous indistinctement» (Platon, Cratyle, 383-384, in Euvres complètes I, Paris, Gallimard, Bibliothèque de la Pléiade, 1984).

${ }^{20}$ Sur la valorisation du mot, en particulier du nom, dans les pratiques mimologistes, voir Genette (op. cit., pp.263-264).

${ }^{21}$ «Terme employé improprement pour désigner le souffle expiratoire qui accompagne l'émission de certains phonèmes » (Martinet, cité par le TLFI).
} 
est conforme à l'objet qu'il désigne non à cause de propriétés inhérentes au signe, mais en raison de caractéristiques de l'objet même qu'il a charge de désigner. Là réside l'artifice, là est le mirage où se rejoignent le «mot en formation» et l'être en formation, où les «aspirations molles» de la diction se doublent du désir d'être. Dans la suite du poème s'esquisse un autre équilibre, précisément entre l'être et l'être en formation. La phrase «le temps le tient dans sa mire » exploite ludiquement l'équivalence. L'être est toujours, chez François Jacqmin, «dans [1] a mire » du temps, avec toute la connotation de violence que recèle l'expression. Dans Le Domino gris, par exemple, le poète notera : "Nous trébuchons sur le temps dès que nous voulons être. // Il n'est jamais l'heure de vivre sans faillir ». Si l'être est aux prises avec le devenir, «l'œuf» pour sa part ne se conçoit que dans une évolution constante, jusqu'à l'éclosion. Son existence est finie, délimitée («Le temps le tient dans sa mire ») et observable dans ... un mire-œufs. Ce qu'a de tragique l'idée de l'être - et la « cassure » de l'accession à l'être - s'atténue dans un jeu de mot qui procède de l' "astuce » généralisée du texte. Ce n'est pourtant justement pas dans cette «astuce » que réside l'ironie du poème - car chaque tentative cratyliste relève toujours d' "une astuce », où l'écrivain peut tout se permettre. Elle apparaît plutôt dans sa mention, dans la dénonciation du processus comme «astuce»- de l' «expression », selon une terminologie à nouveau ambivalente. L'effet de moquerie se renforce par la rupture de ton entre les deux membres distincts de la clausule, depuis la «première tentative d'être » jusqu'au «visage de poussin ».

Dans un poème de «L'été », François Jacqmin paraît amorcer beaucoup plus ostensiblement une (re)motivation poétique du langage :

L'œil s'émiette dans le bouleau.

L'espace frise la folie lorsqu'il traverse son feuillage.

Je m'arrête à temps.

Je pressentais une application personnelle et désastreuse de ce frémissement ${ }^{22}$.

L'allitération des consonnes sifflantes et fricatives dans la deuxième phrase du poème renvoie à une pratique d'écriture connue, sinon conventionnelle, qui est celle de l'harmonie imitative. Par ce biais, le poète tente d'agir sur la langue pour lui conférer une vertu mimétique qu'elle n'a pas. En d'autres termes, il s'efforce d'établir une harmonie entre les sonorités d'une séquence textuelle - de vers ou de prose - et le sens qu'elle détient. Dans Les Saisons, le recours à l'harmonie imitative contreviendrait à l'intégrité de l'univers évoqué, le trahirait par la facticité de l'entreprise. Il ferait entorse à la poétique de la précaution et de la parcimonie qui a été celle de François Jacqmin depuis les premiers mots du premier poème ( « Ce qu'il y a dire du printemps, le printemps le dit») jusqu'à d'autres plus tardifs («J'entends l'arbre exalter l'économie de l'expression »), voire jusqu'aux derniers moments de la dernière saison du cycle ( «Façonner une parole est une épreuve qui revient désormais au sculpteur ${ }^{23} \gg$ ). Enfin, il prêterait le flanc à une ironie extrêmement directe, dont les mécanismes se dévoilent sans ambages. Après avoir fait mine de viser lui aussi à l'harmonie imitative, le poète se rétracte, se dédit. La prise de distance est radicalisée par la scission manifeste des deux énonciations. L'énonciation première, critique, se dissocie brusquement de celle qu'elle enchâsse par l'émergence répétée - mais rarissime dans Les Saisons - du «je », lequel s'efforce, de cette façon, d'échapper à la responsabilité du « désastre » mimologique. De fait, l'ironie ne frappe pas en vain, tant il est vrai qu'eût été «désastreuse »l' «application personnelle »du «frémissement ». Dans la première phrase, c'est effectivement d'un regard qu'il est question, d'une vision. Il n'est rien ici qui s'entende, rien qui nécessite la quête hasardeuse de l'harmonie du son et du sens.

\footnotetext{
${ }^{22}$ Les Saisons, op. cit., p.74.

${ }^{23}$ Les Saisons, op. cit., successivement p.6, p.107 et p.157.
} 
S'il s'avère généralement critique, ironiquement dégradé, l'exercice de la (re)motivation poétique n'en est pas moins, chez François Jacqmin, polysémique, sinon ambigu. Savoir qu'il puisse revenir à un poète de s'interroger sur l'arbitraire du signe, d'y faire même allusion dans un poème - fût-ce de manière moqueuse, ostentatoirement bancale -, constituerait déjà une part d'acquiescement. Le mimologisme ironique de François Jacqmin n'est en cela pas différent de la pratique de l'ironie en général: ce qui est ironisé n'est pas nié, mais simplement mis à distance, dans un jeu qui n'est pas exempt de duplicité. Ce n'est dès lors nullement un hasard, chez un poète à ce point conscient des enjeux langagiers, si un poème met en scène la duplicité même :

La vie reprend haleine dans le lilas.

L'illusion est délectable.

On se protège du destin en mangeant une fraise.

Dans sa douce duplicité, le coucou tente un timide avertissement ${ }^{24}$.

Le poème de «l'illusion délectable » occupe une place significative dans le recueil des Saisons. Il vient après une texte qui s'interroge sur la pertinence du nom : «Peut-on désigner par un mot ce qui ne porte pas de robe ? »; et il est situé avant le poème, déjà cité, qui feint de prétendre justifier le nom par le recours à des pratiques mimologiques : "Dans toutes les langues du monde, l'œuf est un mot en formation ${ }^{25} »$. Maître en concision, François Jacqmin n'a pas besoin de beaucoup plus d'un syntagme - celui de la «douce duplicité » du « coucou »- pour signifier la polysémie du discours mimologique. En effet, d'un point de vue superficiel et littéral, le mot «coucou » trahit déjà une certaine duplicité dans la mesure où il est double, où il est la réduplication d'une seule et même syllabe. Il le fait également en raison de son origine onomatopéique. Davantage qu'aucun vocable, l'onomatopée semble conforme à l'être ou à l'objet qu'elle désigne, essentiellement parce qu'elle entretient avec celui-ci une relation métonymique, telle que, dans le cas présent, le sifflement pour l'oiseau siffleur. Pourtant, même l'onomatopée s'inscrit dans la duplicité - duperie et dualité mimologique, puisqu'elle ne procède en réalité que d'une interprétation phonématique de sons ou de groupes de sons étrangers à notre système articulatoire. Enfin, il n'est pas nécessaire d'insister sur la «duplicité morale » de l'oiseau cette fois, dont le parasitisme est le trait le plus connu. D'autre part, «la douce duplicité » ne constitue pas uniquement une qualification adéquate du «coucou »- du signifiant comme du signifié -; elle en est la réplique mimétique partielle. La réitération des consonnes dentales dans « douce duplicité » fait écho à la répétition des consonnes vélaires du «coucou», tandis que la paire de voyelles vélaires $[\mathrm{u}]$ et $[\ddot{u}]$ n'est guère éloignée, à un niveau infraphonématique, de la reprise de $[u]$ dans le même nom d'oiseau. C'est par conséquent en tous sens et sur tous les plans de l'expression que la «douce duplicité » rendrait compte du «coucou»; et le poème ne parlerait pas seulement du monde - d'une fleur, d'une bestiole et d'un moment d'une saison -, mais de lui-même, de la douce et délectable - mais non moins équivoque - illusion qui le fonde. Nous en fûmes, il est vrai, timidement averti.

\section{Auto-ironie}

S'il est une autre question fondamentale où l'auteur des Saisons court le risque de la contradiction, c'est évidemment celle de la métaphore. Parmi les poètes francophones qui composent leur œuvre après 1945 - soit après les plus belles heures du surréalisme -, François Jacqmin n'est certes pas le seul à manifester une défiance à l'égard de la métaphore

\footnotetext{
${ }^{24}$ Les Saisons, op. cit., p.22.

${ }^{25}$ Les Saisons, op. cit., respectivement p.21 et p.23.
} 
tout en y recourant fréquemment ${ }^{26}$. Il se distingue cependant de deux façons. Tout d'abord, le procès qu'il fait à la métaphore, parangon de tout effort au style, se révèle d'une extrême violence. Les exemples abondent, comme dans ce poème du Livre de la neige, où réapparaissent les « scélérats », déjà rencontrés dans l' «Art poétique » :

\author{
En poésie \\ comme en tout autre domaine, ceux qui sont \\ sans honneur \\ réussissent dans leur entreprise. \\ À défaut d'accéder au sublime, les fourbes \\ se cantonnent dans l'illisible. Leur \\ complication langagière emprunte au monde \\ des scélérats. \\ La métaphore et l'inconduite partagent la même \\ racine $^{27}$.
}

« Complication langagière » des «fourbes », de « ceux qui sont sans honneur », la métaphore n'est pas absente du poème, singulièrement de sa dernière phrase. François Jacqmin n'ignore pas que la recherche de pureté - morale ou esthétique - est toujours vaine, ni qu'avec ses rejets radicaux de la métaphore il frise à tout moment le flagrant délit d'écriture. C'est à nouveau par l'ironie - et par l'auto-ironie - qu'il évite l'écueil menaçant les poètes du blanc et de la retenue, à savoir le bavardage sur le silence, les fleurs de rhétorique sur le refus de la rhétorique :
La neige est partout, et sa douceur désespère
les orateurs sacrés.
Il n'est plus un seul lieu où l'on puisse
placer une métaphore.
Son art
est si pur qu'il n'engendre pas la souffrance
d'une conviction.
L'ouïe est son verbe. C'est dans le clocher sans fin
de sa blancheur
que tintent mes plus beaux sous-entendus ${ }^{28}$.

À la fin du poème, le terme de «sous-entendus» se lit comme l'indice d'une stratégie textuelle à découvrir. A priori, le texte paraît reproduire un type de discours non pas convenu mais souvent exprimé par François Jacqmin - en particulier dans la section «L'hiver » des Saisons et dans Le Livre de la neige -, où «la neige », entre silence et néant, apporte «douceur» et «blancheur» en résistance à l'outrecuidance du moindre propos et à la virulence des « orateurs ». La neige s'y révère comme négation de l'art, comme absence de la moindre expression. «L'ouïe est son verbe » car s'y éteint toute « $\operatorname{ardeur}^{29}$ » poétique : face à l'immensité enneigée, il n'y a rien à dire, il ne faut pas parler, pas écrire, il faut juste « entendre »- en tous sens, percevoir le silence et comprendre, en particulier comprendre que

\footnotetext{
${ }^{26}$ On pensera notamment au poète suisse Philippe Jaccottet. Voir à ce propos l'article de Michèle Monte, «La métaphore dans l'œuvre de Philippe Jaccottet. Entre exhibition et amuïssement », in Pierre Jourde et al. (dir.), Présence de Jaccottet. Paris, Éditions Kimé, 2007, pp.203-225.

${ }^{27}$ François Jacqmin, Le Livre de la neige. Paris, Éditions de la Différence, 1990, p.17.

${ }^{28}$ Le Livre de la neige, op. cit., p.25.

${ }^{29}$ Voir dans Les Saisons : «Qui veut connaître la neige doit retenir son haleine et devenir exsangue comme l'immensité.// La main qui l'examine doit être au degré zéro de l'ardeur.// Ici, la seule faculté requise est l'inaptitude à la rhétorique. » (op. cit., p.145).
} 
le poème invite le lecteur à percer ses secrets, à décrypter ses «sous-entendus », et que parmi ces derniers figure la rédhibitoire incapacité du poète à échapper aux mots. De fait, les «sousentendus» ne ponctuent pas seulement la métaphore filée du «clocher sans fin de la blancheur »; ils en dénoncent également la présence. La métaphore ne se lit plus comme ornement, comme «enluminure ${ }^{30} »$ distraitement concédée - alors qu' «il n'est plus un seul lieu où l'on puisse placer une métaphore »-, mais comme signal de l'ornement, clin d'œil ironique par lequel le poète réussit à ne pas être la dupe de ses refus. Intégrant ironiquement la métaphore dans la critique de la métaphore, François Jacqmin n'est pas loin de se moquer de lui-même. Une posture auto-ironique est atteinte dans un texte qui, à dessein, ne gomme rien du travail rhétorique qui a permis sa composition:

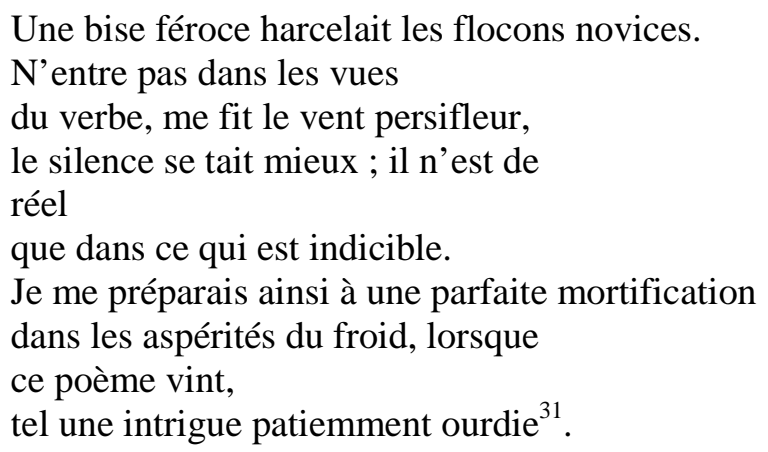

Le poète s'est littéralement soumis au[x] «temps de la fable ${ }^{32} »-$ en l'occurrence, les temps de la narration que sont l'imparfait et le passé simple. De la fable, il manipule ostensiblement les codes, prêtant aux éléments naturels des qualités morales et permettant au vent - ou à la bise - de «harcel[er]» et de parler, selon un processus classique de personnification. Le conseil donné par le vent reprend une idée émise dans de nombreux textes programmatiques de François Jacqmin : «N'entre pas dans les vues/du verbe ». Sa validité est cependant mise en cause dès l'énonciation de sa justification, laquelle prend la forme d'une tautologie : «le silence se tait mieux ». Effectivement, la seule phrase qui s'approcherait du silence, la seule phrase qui ne heurterait pas le silence par un dispositif rhétorique quelconque serait une tautologie $^{33}$. Engager au silence, c'est déjà parler; et la présence de la tautologie ne fait qu'accentuer la contradiction entre le conseil du vent et sa mise en forme poétique. La fable peut asséner des vérités sur les sujets les plus divers, elle peut délivrer des morales de toute sorte ; mais, de par la lourdeur même de son dispositif rhétorique, elle ne peut guère inciter au silence, elle ne le peut en tous cas sans que l'ironie s'en mêle. Le poème du « vent persifleur » ne s'est toutefois pas arrêté à l'ironie involontaire d'un verbe qui se dénonce lui-même - et, partant, sape aussitôt sa propre dénonciation. N'hésitant pas à se prendre pour cible, l'énonciateur mentionne finalement l' « intrigue » qui a présidé à l'élaboration du « poème » et

\footnotetext{
${ }^{30}$ François Jacqmin a publié en 1981 dix poèmes en prose sous le titre oxymorique «Les Enluminures grises 》 (Aa Revue, n 127-128. 19 janvier 1981, pp. 3-14). Certains textes furent repris, réécrits et épurés, dans $L e$ Domino gris, dont celui-ci : «Il faut se garder de dénigrer ce qui est sans expression.// L’inconnu y engrange ses enfants.// En outre, il n'est pas contraire au bon goût de demeurer éperdument sans métaphore. » (p.10).

${ }^{31}$ Le Livre de la neige, op. cit., p.80.

${ }^{32}$ Voir dans Le Livre de la neige (op. cit., p.99) : «Nous conjuguons comme des diables, et prétendons/ construire un temps nouveau à l'aide du temps de la fable. »

${ }^{33}$ Voir cet autre passage du Livre de la neige (op. cit., p.55) : «Je m'emploie/ à réduire l'écart entre la blancheur et la blancheur./ En vain ! La neige est tombée/ en même temps que la neige, et mon identité/ en fut décolorée. » Voir aussi cette déclaration du Poème exacerbé (op. cit., pp.77-78) : «La tautologie, c'est-à-dire l'affirmation répétitive, la confirmation insécable, le retour du même au même, l'infatigable évidence qui provoque à la fois l'espoir et le désespoir de l'intelligence, la tautologie, dis-je, est un des ressorts les plus puissants de ma poésie. Chaque poème est guetté par la tentation tautologique. »
} 
qu'il a lui-même «ourdie » afin que se déploie le poème qu'on vient de lire ${ }^{34}$. On n'y cherchera naturellement pas de velléité de «mortification ». L'auto-ironie n'est en effet pas moins polysémique que l'ironie: s'il se raille lui-même, l'énonciateur affiche surtout ses propres ambiguïtés et s'en fait le régisseur subtil.

\section{Limite de l'ironie}

La tautologie n'est pas seulement pour François Jacqmin un moyen de mise à plat du langage. Elle n'est pas qu'une manière de degré zéro de la pensée et de l'écriture - une phrase parfaite puisque forcément vraie et puisque dépourvue de toute gesticulation, de toute grimace rhétorique. Dans ses poèmes, toute phrase qui s'apparente à une tautologie doit également se lire comme la reprise de l' «atterrante tautologie » ontologique qui énonce que «l'être c'est l'être ${ }^{35} »$ :

La contradiction entre la neige et la neige

atteint

son apogée dans ce qui sépare l'être

de l'être ${ }^{36}$. [...]

Nombre de poèmes portent la trace de ce que François Jacqmin nomme, dans Le Poème exacerbé, sa « cohabitation philosophique ». Les mots ne sont pas innocents : c'est bien d'une « cohabitation» qu'il s'agit, de la longue présence à ses côtés d'un émoi, d'une «pulsion philosophique », «qui ne se distinguait pas radicalement de l'émotion poétique ${ }^{37}$ ». Son intérêt pour la philosophie n'a rien d'intellectuel ni de systématique; ce n'est pas la quête d'un savoir, ni la recherche de réponses à des questions précises. Ce n'est même pas une démarche à proprement parler, mais une étrange passion pour la «folie de l'unique », la mise en mouvement du «mécanisme de l' $\operatorname{extase}^{38} »$ :

Pendant un temps considérable, et parallèlement à l'écriture poétique, je me suis infatué de cette discipline infiniment infructueuse que l'on nomme l'ontologie, c'est-à-dire l'étude de l'être en tant que tel. [...] Nul ne peut évaluer les ravages de l'ontologie s'il n'a succombé à cette barbarie boréale, à cette hypothèse sublimée.

Se laisser toucher par l' «exaltante brûlure »de l'être ${ }^{39}$, c'est prendre la route pour n'aller nulle part, c'est vouer sa pensée et son écriture à l'échec - défaite sublime et ambiguë qui fait dire à l'auteur que c'est « cet échec-là [qu'il] préfère par dessus tout ${ }^{40}$ », mais aussi qu' «entre la métaphysique et le yaourt, il n'y a pas beaucoup de distance ${ }^{41} »$. De l' «immersion » dans «l'illusion ontologique », il ne reste rien - il ne peut rien rester -, hormis un journal

\footnotetext{
${ }^{34}$ On trouvera une confirmation de semblables complots poétiques dans cet aveu tiré à nouveau du Poème exacerbé (op. cit., p.77): «Je me trouve à l'aise lorsqu'un poème se rapproche de la fable, de l'historiette, du conte. [...] Au reste, il m'est habituel de commencer la rédaction d'un poème par le dernier vers, puis, de proche en proche, de remonter vers la première ligne. »

35 « Au terme d'une grande et sinistre histoire d'amour ontologique, Martin Heidegger s'en sortira par cette atterrante tautologie, à savoir que "l'être c'est l'être" » (Le poème exacerbé, op. cit., p.39).

${ }^{36}$ Le Livre de la neige, op. cit., p.82.

${ }^{37}$ Le poème exacerbé, op. cit., p.39.

${ }^{38}$ Le poème exacerbé, op. cit., p.42.

${ }^{39}$ Ibid.

${ }^{40}$ Le poème exacerbé, op. cit., p.51.

${ }^{41}$ Propos exprimé lors d'un entretien avec Pascal Goffaux datant de 1990 (Parole gelée, op. cit., p.25). François Jacqmin devait tenir à la formule car il me la répéta lors d'une de nos rencontres en 1990-1991.
} 
philosophique inédit et «quelques épaves, des pages [qui] ont échoué sur le sable ${ }^{42}$ ». Récusant une approche technicienne ou historienne de l'ontologie et des textes sur l'ontologie, François Jacqmin s'est trouvé des affinités avec la pensée des philosophes présocratiques. S'il apprécie leur manière de «philosopher par fragments ${ }^{43}$ », il considère également que la pensée de l'être n'a sans doute pas vraiment évolué depuis Parménide. L'érudition philosophique serait toujours peu ou prou chimérique, et aucun savant philosophe n'en saurait beaucoup plus que l'Éléate ${ }^{44}$. Aussi certains poèmes très courts semblent-ils reproduire avec fluidité la pensée - combien fragmentaire et énigmatique - du philosophe grec :
Il faut,
littéralement,
n'avoir pas été,
pour être ${ }^{45}$.

François Jacqmin pose à son tour les termes de la confrontation de l'être au devenir, qui est à la base du questionnement ontologique même. Son intervention est minimale, mais lourde d'implications. Parménide était poète, et c'est en poète que Jacqmin écrit - non en penseur épris d'ontologie. De fait, le poème est un quatrain, dont la longueur des vers (2-5-5-2) confère à l'ensemble une structure en miroir. Les trois premiers vers sont séparés de leur suivant par une virgule, pause syntaxiquement non nécessaire mais qui conforte le rythme du texte et invite à s'arrêter presque à chaque mot. Le commentaire du poète tient dans l'adverbe, «littéralement», insistante précision qui devrait reconduire le lecteur à la source parménidienne ${ }^{46}$ - ou du moins l'avertir qu'il n'est pas en présence d'un paradoxe bénin.

Un quatrain extrait d'un livre d'artistes intitulé $\hat{E} t r e^{47}$ semble une variante du poème précédent :
Seul
ce qui est insoluble
est susceptible
d'être.

Ce qui est «insoluble », c'est ce qui ne peut être dissous, donc ce qui ne devient pas, ce qui demeure. Mais c'est aussi, évidemment, ce qui ne peut être résolu, ce qui ne trouvera jamais de solution. En jouant sur la polysémie du mot «insoluble», François Jacqmin recourt à nouveau au processus de double énonciation, par lequel la pensée de l'être s'énonce dans le même temps qu'elle se voit dénoncée comme aporie. Le texte n'est toutefois qu'à peine

\footnotetext{
${ }^{42}$ Le poème exacerbé, op. cit., pp.55, 56 et 51.

${ }^{43}$ Le poème exacerbé, op. cit., p.36.

${ }^{44}$ Cela aussi évidemment est sujet à caution, puisque notre connaissance des présocratiques est elle-même particulièrement labile, sinon douteuse. Dans un ouvrage récent, Jean Bollack a montré que la connaissance que les contemporains pouvaient avoir des fragments du poème de Parménide était largement tributaire des lectures trop exclusivement ontologiques produites par Platon et, bien plus tard, par Heidegger (Parménide, de l'étant au monde. Lagrasse, Éditions Verdier, coll. Verdier/Poche, 2006).

${ }^{45}$ Poème extrait du recueil Particules, publié une première fois au Daily-Bul en 1981 (La Rose de décembre et autres poèmes, op. cit., p.91).

${ }^{46} \mathrm{Ou}$, de nouveau, à ce qu'il croit savoir du texte de Parménide. On pourra rapprocher le quatrain de François Jacqmin du fragment 6 du poème de Parménide : «Ce qui est utile, c'est de dire que ceci est étant et de penser ainsi ceci ; car il y a moyen qu'il soit; pour le rien, il n'y a pas moyen qu'il soit; ces choses en tout cas je t'enjoins, moi, de te les expliquer à toi-même. » (trad. Bollack, op. cit., p.121).

${ }^{47}$ François Jacqmin, Être. Nantes, Cahiers du Pré Nian, 1984. Recueil non paginé. Sérigraphies de Bertrand Bracaval, Guy Boulay et Jean-Luc Herman.
} 
ironique : il ne moquerait rien ni personne ; il rappellerait plutôt - dans un chef-d'œuvre de concision - qu'il y a, effectivement, des chemins qui ne mènent nulle part. Du reste, c'est toujours avec un discours, avec un texte, que l'ironie, figure métacommunicationnelle, prend ses distances. Face à l'être - qui n'est pas le dire sur l'être -, comme face à la beauté de la nature $^{48}$, l'ironiste est forcé de s'arrêter, et le poète de se « déclarer démuni ${ }^{49}$ ».

\section{Par ailleurs, comique}

Le comique et l'ironie ne sont pour François Jacqmin que des degrés différents d'une même réalité. L'ironie ne s'oppose pas chez lui au comique ${ }^{50}$ : elle est l'intensité de comique qu'il peut atteindre; elle en est la manifestation «souterraine», « oblique ${ }^{51}$ », discrète. Et elle recèle un mérite majeur : elle le protège à de multiples titres et de multiples façons - contre l'illusion où se perd irrémédiablement l'homme qui parle, l'homme qui écrit, a fortiori l'homme qui écrit en se croyant poète ou écrivain ; mais aussi contre le silence, la tentation de l'abstention, voire le rejet dogmatique - et fatalement paradoxal - de l'illusion représentative. Dans Le poème exacerbé, lorsqu'il veut donner des exemples de créations «enrobées d'humour », l'auteur cite «deux poèmes un peu bouffons sur les escargots »: "L'escargot fait le bonheur des portraitistes. Il ne bouge pas. On peut le dessiner à loisir, sans craindre de perdre un détail, une expression ${ }^{52}$. [...]». La bouffonnerie de ces textes pourra se juger pour le moins timide : c'est qu'il ne faut pas craindre de s'aventurer dans les marges du corpus poétique pour trouver des productions incongrues où François Jacqmin se laisse aller plus ouvertement à l'absurde et au non sense. L'iconographie a pu exploiter cette veine, grâce à laquelle le poète parvient à écorner son image de gentleman poli, distingué, so british. Dans les années soixante, François Jacqmin fut photographié par Georges Thiry. Il porte veston, cravate et gilet. Il est debout devant une bibliothèque et tient dans la main gauche un improbable pot de fleur. C'est le triple portrait pince-sans-rire d'un écrivain, d'un botaniste et d'un farceur ${ }^{3}$. C'est encore le farceur qui admet, beaucoup plus tard, de se faire photographier avec une casserole sur la tête ${ }^{54}$. Qu'il se prétende - probablement avec raison «le membre le plus tranquille de la Belgique Sauvage ${ }^{55}$ » ne lui interdit pas pour autant la pitrerie occasionnelle. Aussi n'est-ce guère non plus un hasard s'il a traduit de l'anglais, avec Richard Tialans, une oeuvre de l'artiste et poète néo-dadaïste Robert Filliou. Idiot-ci, Idiot-

\footnotetext{
${ }^{48}$ Voir cette réflexion qui amorce la conclusion sur la «cohabitation philosophique » (Le poème exacerbé, op. cit., p.58) : «Au printemps, la terre s'annonce dans un tonnerre de fleurs, elle s'apprête à couvrir le sol de son énorme illusion, et à répandre son parfum entêtant dans l'air. Je reste béat d'admiration et de crainte. Je ne cherche pas à comprendre ; je demeure perpétuellement au stade infantile de l'étonnement : j'ai reçu la fixité en partage. »

${ }^{49}$ Le Livre de la neige, op. cit., p.55.

${ }^{50}$ Cette absence de distinction ne va pas forcément de soi. Hegel établit une différence en fonction des « contenu[s]» que l'ironie et le comique détruisent. «Le comique » se bornerait à « démolir ce qui est dépourvu de valeur en soi, un phénomène faux et contradictoire, une lubie, une manie... », alors que l'ironie «consiste dans l'auto-destruction de ce qui est noble, grand, parfait » (Esthétique. Tome I. Paris, Aubier-Montaigne, 1944, pp.92-93). De son côté, le poète Edmond Jabès joue sur une distinction formelle : «L'humour est poésie. Le comique est prose. » (Le Seuil Le Sable. Poésies complètes 1943-1988. Paris, Gallimard, coll. Poésie Gallimard, 1990, p.168). Ces distinguos paraissent sans objet en ce qui concerne François Jacqmin.

${ }^{51}$ Selon l'expression de Philippe Hamon, qui voit justement dans l'ironie une « écriture oblique ».

${ }^{52}$ Le poème exacerbé, op. cit., pp.25-26.

${ }^{53}$ La photo est reprise dans la réédition des Saisons (Bruxelles, Éditions Labor, coll. Espace Nord n50, 1988).

${ }^{54}$ Photo de Michel Duez en illustration de l'article cité de Martine Gerardy.

${ }^{55}$ Le poème exacerbé, op. cit., p.9.
} 
là ${ }^{56}$ (Ample food for stupid thought) est une suite de 90 questions qui parfaitement logiques, qui parfaitement absurdes, qui d'une banalité confinant à la sagesse : «Préféreriez-vous une vie de chien ?// Pourquoi vous êtes-vous levé ce matin ?// Combien en faudrait-il pour que ce soit trop ?// [...] // Qui dit que les rats sont gentils ?// [...] // Êtes-vous génial ?// Qu'aimeriezvous faire à votre facteur ? »C'est drôle, impertinent, parfois un peu inquiétant - car il est des questions que l'on préfèrerait éviter - et, selon les points de vue, profond comme un traité de métaphysique ou comme un pot de yaourt.

\footnotetext{
${ }^{56}$ Robert Filliou, Idiot-ci, Idiot-là. Liège, AA Éditions - Yellow Now, 1977. Traduction et adaptation française par François Jacqmin et Richard Tialans. L'ouvrage se présente sous la forme d'un recueil de 90 cartes postales contenant chacune une question différente.
} 